OPEN ACCESS

Edited by:

Antonella D'Amico,

University of Palermo, Italy

Reviewed by:

Wiktor Sieklicki,

Gdansk University of Technology,

Poland

Chung Hyuk Park,

George Washington University,

United States

*Correspondence:

Hongwu Wang

hongwu.wang@phhp.ufl.edu

Specialty section:

This article was submitted to

Human-Robot Interaction,

a section of the journal

Frontiers in Robotics and Al

Received: 30 October 2021

Accepted: 25 January 2022

Published: 25 February 2022

Citation:

Chandrashekhar $R$, Wang $H$,

Rippetoe J, James SA, Fagg AH and

Kolobe THA (2022) The Impact of

Cognition on Motor Learning and Skill Acquisition Using a Robot Intervention

in Infants With Cerebral Palsy.

Front. Robot. Al 9:805258.

doi: 10.3389/frobt.2022.805258

\section{The Impact of Cognition on Motor Learning and Skill Acquisition Using a Robot Intervention in Infants With Cerebral Palsy}

\author{
Raghuveer Chandrashekhar ${ }^{1}$, Hongwu Wang ${ }^{1 *}$, Josiah Rippetoe ${ }^{2}$, Shirley A. James ${ }^{2}$, \\ Andrew H. Fagg ${ }^{3,4}$ and Thubi H. A. Kolobe ${ }^{2}$
}

${ }^{1}$ Department of Occupational Therapy, College of Public Health and Health Professions, University of Florida, Gainesville, FL, United States, ${ }^{2}$ Department of Rehabilitation Sciences, College of Allied Health, University of Oklahoma Health Sciences Center, Oklahoma City, OK, United States, ${ }^{3}$ Department of Computer Science, University of Oklahoma, Norman, OK, United States, ${ }^{4}$ Institute of Biomedical Engineering, Science, and Technology, University of Oklahoma, Norman, OK, United States

Background: Cerebral Palsy $(\mathrm{CP})$ is a neurodevelopmental disorder that encompasses multiple neurological disorders that appear in infancy or early childhood and persist through the lifespan of the individual. Early interventions for infants with CP utilizing assisted-motion robotic devices have shown promising effects in rehabilitation of the motor function skills. The impact of cognitive function during motor learning and skill acquisition in infants using robotic technologies is unclear.

Purpose: To assess the impact of cognitive function of infants with and without CP on their motor learning using the Self-Initiated Prone Progression Crawler (SIPPC) robot.

Methods: Statistical analysis was conducted on the data obtained from a randomized control trial in which the movement learning strategies in infants with or at risk for CP was assessed during a 16-week SIPPC robot intervention. Cognitive function was measured by the Bayley scales of Infant and Toddler Development-Third edition (Bayley-III) and motor function was measured by the Movement Observation Coding Scheme (MOCS). The infants were categorized into three distinct groups based on their cognitive scores at baseline: "above average" ( $\left.n_{1}=11\right)$, "below average" $\left(n_{2}=10\right)$, and "average" $\left(n_{3}=26\right)$. Triweekly averages of the MOCS scores (observations at five time points) were used for the analyses. This study involved computing descriptive statistics, data visualization, repeated measures analysis of variances (rmANOVA), and survival analyses.

Results: The descriptive statistics were calculated for the MOCS and Bayley III scores. The repeated measures ANOVAs revealed that there was a statistically significant effect of time $(p<0.0001)$ on scores of all subscales of the MOCS. A statistically significant effect of interaction between group and time $(p<0.05)$ was found in MOCS scores of subscales 1 and 2. The survival analyses indicated that infants in different cognition groups significantly differed $(p<0.0001)$ in their ability to achieve the crawling milestone within the 16 -week intervention period. 
Conclusion: The findings in this study reveal the key movement strategies required to move the SIPPC robot, assessed by the MOCS, vary depending on the infants' cognition. The SIPPC robot is well-matched to cognitive ability of infants with CP. However, lower cognitive ability was related to delayed improvement in their motor skills.

Keywords: cognition, robot, human robot interaction interface, motor learning, cerebral palsy

\section{BACKGROUND AND SIGNIFICANCE}

Cerebral Palsy (CP) is a neurodevelopmental disorder that encompasses multiple neurological disorders that appear in infancy or early childhood and persist through the lifespan of the individual (Peter, 2007). Population-based studies across the world report prevalence estimates of $\mathrm{CP}$ within the range of 1.5 to more than 4 per 1,000 live births (Arneson et al., 2009; Bhasin et al., 2006; CDCdataforCP, 2020; van Gorp et al., 2020; NINDSWebsite, 2019). The CDC's Autism and Developmental Disabilities Monitoring Network reports one in 323 children in the United States have CP (CDCdataforCP, 2020). In 2000, estimates of lifetime cost encompassing all children born with $\mathrm{CP}$ in the United States was $\$ 11.5$ billion (Honeycutt et al., 2004) and although the prevalence has not changed significantly in the past 10 years, the cost of care associated with $\mathrm{CP}$ has increased (Novak et al., 2013; Oskoui et al., 2013; van Gorp et al., 2020). CP continues to be the most physically disabling condition in the United States and among numerous other complications experienced by adults and children with $\mathrm{CP}$, the most disabling is impaired mobility (Turk, 2009; McAdams and Juul, 2011). Children with CP experience some or all of the following symptoms: poor muscle coordination (ataxia), muscle spasticity, impaired postural control, upper or lower extremity weakness, tremors, delays in reaching motor skill milestones (independent crawling and walking), toe walking, crouched or adducted "scissored" gait, altered muscle tone, excessive drooling, and difficulty swallowing or speaking, and lack of manual dexterity (Oskoui et al., 2013; Turk, 2009; NINDSWebsite, 2019). These impairments impact these children's motor, cognitive, psychological, and social development (HaddersAlgra, 2000; Anderson et al., 2013).

Because CP is caused by an irreversible injury to the brain, there are no currently known cures. Intervention strategies focus on maintaining and improving mobility, quality of life, function, and prevention of secondary complications. Cognitive impairments foster motor and functional impairments, which further exacerbate the cognitive impairment, resulting in a cycle of debilitating symptoms (Monteiro et al., 2010; Robert et al., 2013; Stadskleiv, 2020). Because fundamental functional ability and motor skills develop early within typically developing children, it is critical to initiate intervention early for children/ infants at risk for, or diagnosed with CP (Shonkoff and Meisels, 2000; Bayon et al., 2016). Also essential, is incorporating quality of movement and functional activities within these intervention strategies, while encouraging interaction within a variety of environments to foster comprehensive development (Shonkoff and Meisels, 2000; Bayon et al., 2016; Stadskleiv, 2020). A variety of early intervention strategies are used to address complications associated with $\mathrm{CP}$, and include physical therapy (PT), occupational therapy (OT), oral medication or botulinum toxin pumps or injections for spasticity, orthotics, and surgery. Depending on the type of $\mathrm{CP}$, successful interventions can also include partial bodyweight supported treadmill training, constraint induced movement therapy, and robot assisted therapy (Novak et al., 2013; Robert et al., 2013; Bayon et al., 2016; Sadowska et al., 2020). While the outcomes are inconsistently reported (Novak et al., 2013), the literature does indicate intervention focusing on motor learning, partial bodyweight supported treadmill training, constraint induced movement therapy, and robot assisted therapy are more effective in enhancing functional mobility outcomes (Novak et al., 2013; Bayon et al., 2016; Kolobe and Fagg, 2019).

Among the early interventions involving motor learning, assisted-motion robotic devices such as the CPWalker, PALMIBER vehicle, and Self-Initiated Prone Progression Crawler (SIPPC), have demonstrated promise in rehabilitating functional mobility skills in infants with CP (Raya et al., 2015; Kolobe and Fagg, 2019). However, a common limitation of robotic devices is the complexity of the intervention devices, which makes them difficult to use when the children have cognitive impairments (Hogan and Krebs, 2004; Krebs and Hogan, 2006; Bayon et al., 2016). Since individuals with different levels of cognition learn the same task at varying paces, it is necessary to develop assistive devices that can be used by individuals with dissimilar levels of cognition. The CPWalker is a comprehensive robotic platform that consists of a smart walker with body weight and autonomous locomotion support, a wearable exoskeleton robot, and a motor neuroprosthesis for joint range of motion support, controlled by a multimodal human-robot interface (Raya et al., 2015). The multimodal human-robot interface used to control the CPWalker allows the integration of the peripheral nervous system (PNS) as well as the central nervous system (CNS), thus combining the physical (PNS) and cognitive (CNS) approach to motor rehabilitation in children with CP (Raya et al., 2015). The PALMIBER vehicle is a pre-industrial robotic vehicle designed and developed with an "assist as needed" paradigm and a playful interface. The PALMIBER vehicle promotes interaction between the child with $\mathrm{CP}$ and their environment through mobility experiences. Similar to the CPWalker, the PALMIBER vehicle also integrates a physical and cognitive approach in addressing motor impairments caused by CP (Raya et al., 2015).

The SIPPC robot differs from the CPWalker and the PALMIBER because it allows early intervention using robotics for infants with or at risk of developing CP (Ghazi et al., 2016; Kolobe and Fagg, 2019). Additionally, the SIPPC robot takes advantage of each infant's self-initiated movement, critical for 
early locomotion and crucial for enhancing synaptic connection within the brain during the early stage of development (de Vries and de Groot, 2002; Kolobe and Fagg, 2019). The primary driving forces behind the conceptualization, design, and function of the SIPPC robot are two motor learning mechanisms available within the infant central nervous system: reinforcement learning (RL) and error-based learning (EBL). The SIPPC robot uses a physical and cognitive approach to rehabilitation, however it is uniquely designed to capture and enhance movement effort when infants are developing prone locomotion (crawling), one of the major mobility milestones in infant development (Miller et al., 2015; Raya et al., 2015; Kolobe and Fagg, 2019).

While robotic devices have shown promise in the rehabilitation of children with $\mathrm{CP}$, the impact of cognitive function during motor learning and skill acquisition in infants using robotic technologies is still unclear. Understanding the impact of cognitive function on the outcomes of robotic intervention will guide the design and configuration of the human-robot interface. The aim of this study is to evaluate the effect of cognitive ability of infants with and without CP on their proficiency in learning SIPPC robot mobility (Miller et al., 2015; Ghazi et al., 2016; Kolobe and Fagg, 2019). In addition to informing future revision of the SIPPC robot, findings from this study will illustrate the interplay between robotic movement, learning, and cognition, as well as the levels of end-user cognitive ability necessary for the use of this interface.

\section{METHODS AND MATERIALS}

\section{Participants}

Sixty-three infants between four and 5 months old, with and without $\mathrm{CP}$, were recruited for this study involving the use of the SIPPC robot. Phase I recruited typically developing infants, and phase II recruited infants with or at high risk of developing $\mathrm{CP}$. The inclusion criterion for infants in phase I was a motor development index (MDI) of at least 85 on the Bayley Scales of Infant and Toddler Development ( $3^{\text {rd }}$ ed., Bayley III) or at least a $\mathrm{z}$-score of -1 standard deviation (sd) on the Test of Infant Motor Performance (TIMP) (Campbell et al., 2002; Kolobe et al., 2004). Inclusion criteria for infants in phase II were a TIMP $z$-score of -1 sd or lower, and MDI of 70 or lower, a confirmed diagnosis of $\mathrm{CP}$, or MRI results indicating brain injury, prior to age two (Campbell et al., 2002; Kolobe et al., 2004). Upon entry into the study, parents of eligible infants consented their children and completed the Family Interview Form (FIF). The FIF was designed to collect demographic information and it included parents' level of education, age, occupation, marital status, and household structure. Infant demographics included medical, health, birth, and developmental histories. The family demographic information collected from the FIF was not used in the data analyses as it did not directly measure the infants' cognitive abilities or their motor learning and skill acquisition. The original study was reviewed by the University of Oklahoma IRB \#5120 and the data analysis used de-identified data.

\section{Outcome Measures}

The Bayley Scales of Infant and Toddler Development (3rd ed.) (Bayley, 2006), and the Movement Observation Coding Scheme (MOCS) (Rule, 2010) were the major outcome measures used in this study. The MOCS consists of 42 items and four subscales (Subscale 1: Posture and support, Subscale 2: Exploratory selection and progression, Subscale 3: Mastery of Propulsion, and Subscale 4: Socio-emotional responses). The MOCS measured the motor learning ability of the infants.

\section{Robotic Intervention Protocol}

Infants from both phases completed the same protocol while using the SIPPC robot, involving two training sessions per week, up to 16 weeks, or until the crawling milestone was reached, where the crawling milestone was defined as the ability of the infant to crawl without assistance from the SIPPC robot or any other assistive device. Intervention began when infants reached 5-7 months of age, and occurred at either the infant's home or the Human Development Laboratory at the University of Oklahoma Health Sciences Center. Therapists fitted infants with a securely strapped jumpsuit equipped with Inertial Measurement Unit (IMU) sensors. The intervention protocol involved the following steps:

1. Familiarization with the SIPPC robot-Infants played with both familiar and novel toys while being acclimated to the SIPPC robot for the first 1-2 min.

2. Assisted movement of infant's arms and legs-If the infant was unable to initiate crawling toward a toy, investigators or caregivers moved the infant's arms and legs to allow the infant to understand how to move the robot.

3. Self-initiated mobility-Researchers and caregivers encouraged infants to move the SIPPC using toys as a reward, for up to $5 \mathrm{~min}$. If the infant was unable to move, researchers repeated step 2.

If the infant was able to crawl without assistance at or before the 16 weeks, i.e., if the crawling milestone was achieved, the infant no longer needed assistance from the SIPPC robot, thus concluding their participation in the study. Research staff repeated the Bayley III either when the infant achieved independent crawling, or at the end of 16 weeks.

\section{Data Analysis Procedure}

The two major variables of interest for this data analysis were cognitive ability as measured by the mental development index of the Bayley-III (MDI) and motor function as measured by the MOCS. Infants were categorized into three distinct groups based on their baseline MDI scores: "above average" cognitive ability (group 1), "below average" cognitive ability (group 2), and "average" cognitive ability (group 3). Research staff calculated triweekly average scores on each subscale of the MOCS tool and used these as the outcome/dependent variable for all analyses. We measured change over time using five measurement time points containing the mean tri-weekly MOCS. We used variables indicating the crawling status of the infant and the length of follow-up/duration of intervention to conduct a survival analysis. This analysis allowed us to identify the percentage of infants who 
TABLE 1 | Mean and standard deviation values for demographic and baseline cognitive scores for all infants $(n=47)$, infants in the above average $(n=11)$, below average ( $n=$ $10)$, and average $(n=26)$ cognition groups.

\begin{tabular}{|c|c|c|c|c|}
\hline Demographics/Group & All participants & "Above average" cognitive group & "Below average" cognitive group & "Average" cognitive group \\
\hline $\mathrm{N}$ & 47 & 11 & 10 & 26 \\
\hline Age at baseline (months) & $4.66(0.60)$ & $4.45(0.69)$ & $4.90(0.57)$ & $4.65(0.56)$ \\
\hline Weight (lb) & $3.71(2.27)$ & $3.43(1.25)$ & 4.96 (3.28) & 3.29 (1.93) \\
\hline Gestational age (weeks) & $31.31(5.12)$ & $31.87(3.50)$ & $32.34(6.95)$ & $30.69(4.95)$ \\
\hline Cognitive score (Bayley III) & $99(18.57)$ & 119 (3.93) & $70(14.34)$ & $102(6.19)$ \\
\hline
\end{tabular}

TABLE 2 | MOCS subscale scores for the three cognitive groups at baseline: "above average" cognition group ( $n=11)$, "average" cognition group ( $n=26)$, and "below average" cognition group $(n=10)$.

\begin{tabular}{|c|c|c|c|c|c|c|c|c|c|}
\hline Group (based on cognitive ability) & Mean & Median & Std dev & $N$ & Min. & Max. & Lower $95 \%$ CL for mean & Upper $95 \%$ CL for mean & $p$-Value \\
\hline \multicolumn{10}{|l|}{ Subscale 1: Posture and support } \\
\hline "Above average" cognition & 7.0 & 7.0 & 2.1 & 11 & 3.0 & 10.0 & 5.6 & 8.5 & 0.0523 \\
\hline "Average" cognition & 7.0 & 7.0 & 2.0 & 22 & 3.7 & 10.0 & 6.1 & 7.9 & \\
\hline "Below average" cognition & 5.3 & 5.5 & 1.6 & 10 & 2.3 & 8.0 & 4.1 & 6.4 & \\
\hline \multicolumn{10}{|c|}{ Subscale 2: Exploratory selection and progression } \\
\hline "Above average" cognition & 17.2 & 17.3 & 1.9 & 11 & 13.5 & 20.3 & 15.9 & 18.4 & 0.6819 \\
\hline "Average" cognition & 15.3 & 16.7 & 5.9 & 22 & 3.0 & 25.0 & 12.7 & 17.9 & \\
\hline "Below average" cognition & 15.5 & 14.1 & 8.4 & 10 & 5.5 & 36.0 & 9.5 & 21.5 & \\
\hline \multicolumn{10}{|l|}{ Subscale 3: Mastery of propulsion } \\
\hline "Above average" cognition & 20.8 & 21.0 & 4.2 & 11 & 13.7 & 27.5 & 17.9 & 23.6 & 0.3003 \\
\hline "Average" cognition & 17.2 & 17.8 & 9.9 & 22 & -3.5 & 35.0 & 12.8 & 21.6 & \\
\hline "Below average" cognition & 14.6 & 16.3 & 10.8 & 10 & -5.5 & 28.7 & 6.9 & 22.3 & \\
\hline
\end{tabular}

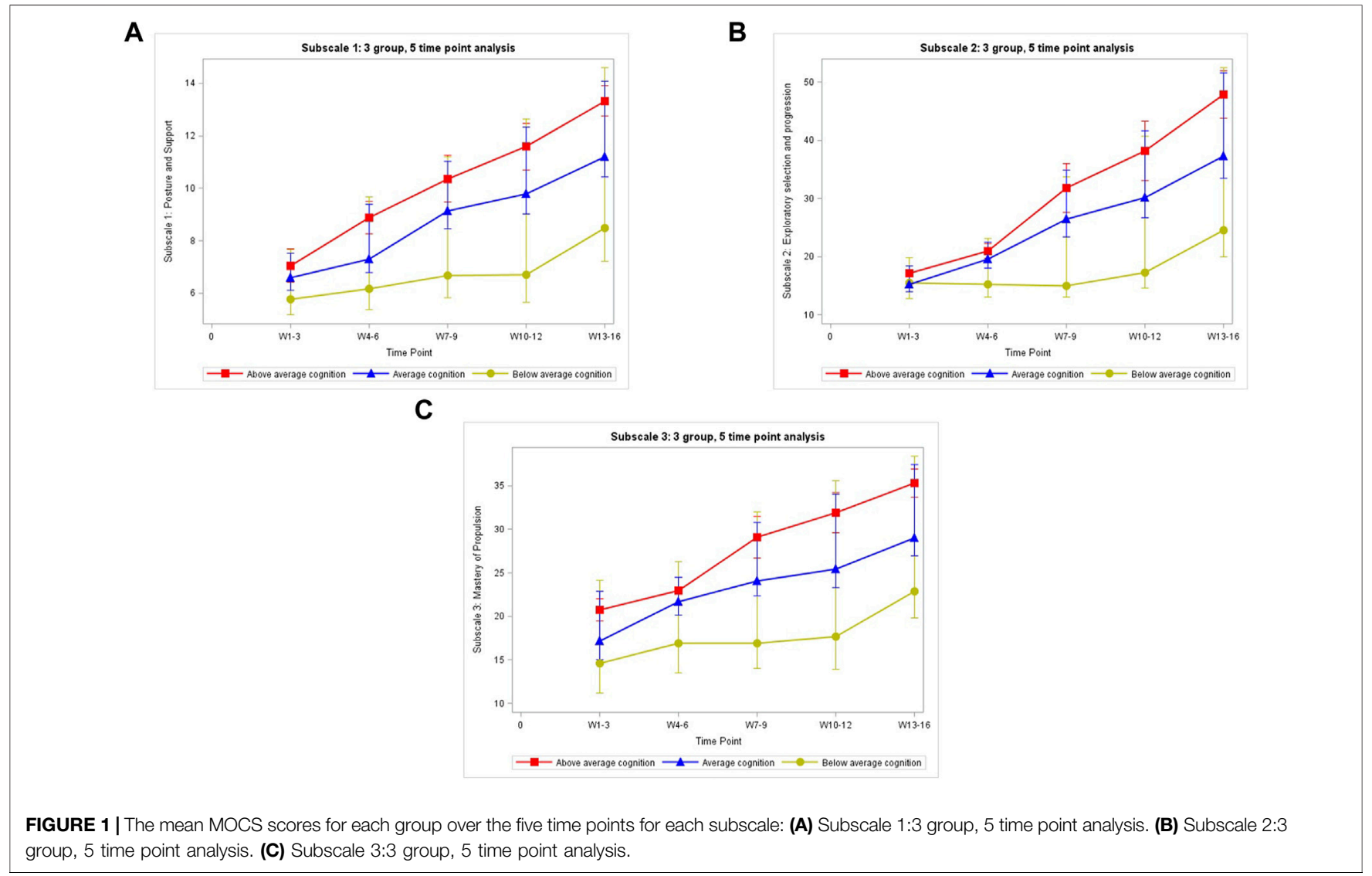


TABLE 3 | Repeated measures ANOVA for each subscale (alpha = 0.05).

Subscale 1: Posture and support

\begin{tabular}{|c|c|c|c|c|c|}
\hline Effect/Time/Group & Standard Error & t Value & $\operatorname{Pr}>|t|$ & Beta Estimates & $95 \% \mathrm{Cl}$ for the Beta Estimates \\
\hline Intercept & 0.4032 & 17.09 & $<0.0001^{*}$ & 6.8731 & $6.0624,7.6838$ \\
\hline \multicolumn{6}{|l|}{ Time 1 (weeks 1-3)-referent time } \\
\hline Time 2 (weeks 4-6) & 0.3498 & 1.52 & 0.1355 & 0.5320 & $-0.1730,1.2369$ \\
\hline Time 3 (weeks 7-9) & 0.5074 & 4.50 & $<0.0001^{*}$ & 2.2840 & $1.2614,3.3065$ \\
\hline Time 4 (weeks 10-12) & 0.6021 & 4.96 & $<0.0001^{*}$ & 2.9865 & $1.7730,4.2000$ \\
\hline Time 5 (weeks 13-160 & 0.6396 & 7.29 & $<0.0001^{\star}$ & 4.6622 & $3.3732,5.9512$ \\
\hline \multicolumn{6}{|c|}{ "Average" cognition group-reference group } \\
\hline "Above average" cognition group & 0.7139 & 0.24 & 0.8103 & 0.1724 & $-1.2665,1.6112$ \\
\hline "Below average" cognition group & 0.7379 & -2.20 & $0.0331^{\star}$ & -1.6231 & $-3.1102,-0.1359$ \\
\hline \multicolumn{6}{|c|}{ Significant interaction between time 2 and "above average" cognition group (group 1) (time*group) } \\
\hline Time $2^{*}$ group 1 & 0.6154 & 2.11 & $0.0401^{*}$ & 1.3014 & $0.0612,2.5416$ \\
\hline
\end{tabular}

Subscale 2: Exploratory selection and progression

\begin{tabular}{|c|c|c|c|c|c|}
\hline Effect/Time/Group & Standard Error & t Value & $\operatorname{Pr}>|t|$ & Beta Estimates & $95 \% \mathrm{Cl}$ for the Beta Estimates \\
\hline Intercept & 1.2068 & 12.33 & $<0.0001^{*}$ & 14.8770 & $12.4449,17.3091$ \\
\hline \multicolumn{6}{|l|}{ Time 1 (weeks 1-3)-reference time } \\
\hline Time 2 (weeks 4-6) & 1.0402 & 4.35 & $<0.0001^{*}$ & 4.5201 & $2.4238,6.6164$ \\
\hline Time 3 (weeks 7-9) & 2.5160 & 4.43 & $<0.0001^{*}$ & 11.1520 & $6.0813,16.2227$ \\
\hline Time 4 (weeks 10-12) & 2.9394 & 5.08 & $<0.0001^{*}$ & 14.9412 & $9.0172,20.8652$ \\
\hline Time 5 (weeks 13-160 & 3.0033 & 7.44 & $<0.0001^{\star}$ & 22.3314 & $16.2785,28.3842$ \\
\hline \multicolumn{6}{|c|}{ "Average" cognition group-reference group } \\
\hline "Above average" cognition group & 2.1506 & 1.06 & 0.2960 & 2.2745 & $-2.0598,6.6088$ \\
\hline "Below average" cognition group & 2.2231 & 0.29 & 0.7749 & 0.6397 & $-3.8406,5.1200$ \\
\hline \multicolumn{6}{|c|}{ Significant interaction between time (time 2, 3, 4, and 5) and "below average" cognition group (group 2) (time*group) } \\
\hline Time 2*group 2 & 1.9063 & -2.49 & $0.0165^{*}$ & -4.7534 & $-8.5954,-0.9114$ \\
\hline Time $3^{*}$ group 2 & 4.7039 & -2.47 & $0.0175^{\star}$ & -11.6187 & $-21.0987,-2.1387$ \\
\hline Time $4^{*}$ group 2 & 5.5326 & -2.38 & $0.0215^{\star}$ & -13.1912 & $-24.3414,-2.0410$ \\
\hline Time $5^{\star}$ group 2 & 5.6720 & -2.34 & $0.0241^{*}$ & -13.2480 & $-24.6792,-1.8169$ \\
\hline \multicolumn{6}{|c|}{ Subscale 3: Mastery of propulsion } \\
\hline Effect/Time/Group & Standard Error & t Value & $\operatorname{Pr}>|t|$ & Beta Estimates & $95 \% \mathrm{Cl}$ for the Beta Estimates \\
\hline Intercept & 1.7146 & 10.30 & $<0.0001^{*}$ & 17.6558 & $14.2002,21.1114$ \\
\hline \multicolumn{6}{|l|}{ Time 1 (weeks 1-3)-reference time } \\
\hline Time 2 (weeks 4-6) & 0.7643 & 4.65 & $<0.0001^{*}$ & 3.5529 & $2.0125,5.0932$ \\
\hline Time 3 (weeks 7-9) & 1.2088 & 5.29 & $<0.0001^{\star}$ & 6.3999 & 3.9637, 8.8362 \\
\hline Time 4 (weeks 10-12) & 1.4358 & 5.61 & $<0.0001^{*}$ & 8.0592 & $5.1656,10.9528$ \\
\hline Time 5 (weeks 13-160 & 1.2790 & 9.38 & $<0.0001^{\star}$ & 12.0018 & $9.4243,14.5794$ \\
\hline \multicolumn{6}{|c|}{ "Average" cognition group-reference group } \\
\hline "Above average" cognition group & 2.6821 & 1.11 & 0.2750 & 2.9649 & $-2.4405,8.3704$ \\
\hline "Below average" cognition group & 2.7743 & -1.98 & $0.0543^{\star \star}$ & -5.4856 & $-11.0768,0.1056$ \\
\hline
\end{tabular}

${ }^{*}$ means statistically significant, i.e., $\mathrm{p}<0.05$; ${ }^{*}$ means trending towards statistical significance.

successfully achieved independent crawling (time to crawl variable), or the end of a 16-week intervention, whichever came first.

\section{Statistical Analysis}

We computed summary statistics for variables within each "cognitive ability" group as well as the entire sample. We utilized repeated measures analysis of variance (repeated measures ANOVA) with the MOCS scores (tri-weekly averages) as the dependent variable, and including both "group" and "time" as independent variables, using an "unstructured" covariance structure. We used survival analysis to determine the crawling status of infants at the end of the study, as wells as their "time to crawl." Finally, survival plot analysis for the first three subscales of the MOCS (Subscale 1: Posture and support, Subscale 2: Exploratory selection and progression, and
Subscale 3: Mastery of Propulsion) allowed us to determine significant differences between the three cognitive groups of infants. Statistical procedures were repeated for all subscales of the MOCS except subscale 4 ("socio-emotional responses") which we excluded from all analyses as this subscale does not provide information about motor learning ability of infants. We plotted mean MOCS scores over the five time points (representing weeks $1-3,4-6,7-9,10-12,13-15)$ for each of the three subscales of the MOCS. All analyses utilized SAS 9.4 (Carey, NJ) with alpha $=0.05$.

\section{RESULTS}

Forty-nine infants completed 16-week videotaped intervention sessions, complete with movement coding. One of these infants 
TABLE 4 | Survival analysis results using time to crawl, including the number of infants in each group who achieved the crawling milestone $(n=47)$.

\begin{tabular}{|c|c|c|c|c|c|}
\hline Group & $N$ & Crawled & $\begin{array}{l}\text { Number who failed to } \\
\text { crawl }\end{array}$ & $\begin{array}{l}\text { Percent who failed to } \\
\text { crawl }\end{array}$ & $\begin{array}{l}\text { Likelihood of achieving crawling milestone within } \\
\qquad 16 \text { weeks }\end{array}$ \\
\hline $\begin{array}{l}\text { "Above average" cognition } \\
\text { group }\end{array}$ & 11 & 11 & 0 & 0.00 & $1.48(1.23,1.77)$ \\
\hline $\begin{array}{l}\text { "Below average" cognition } \\
\text { group }\end{array}$ & 10 & 3 & 7 & 70.00 & $0.28(0.20,0.37)$ \\
\hline "Average" cognition group & 26 & 21 & 5 & 19.23 & Reference group \\
\hline Total & 47 & 35 & 12 & 25.53 & NA \\
\hline
\end{tabular}

\section{A}

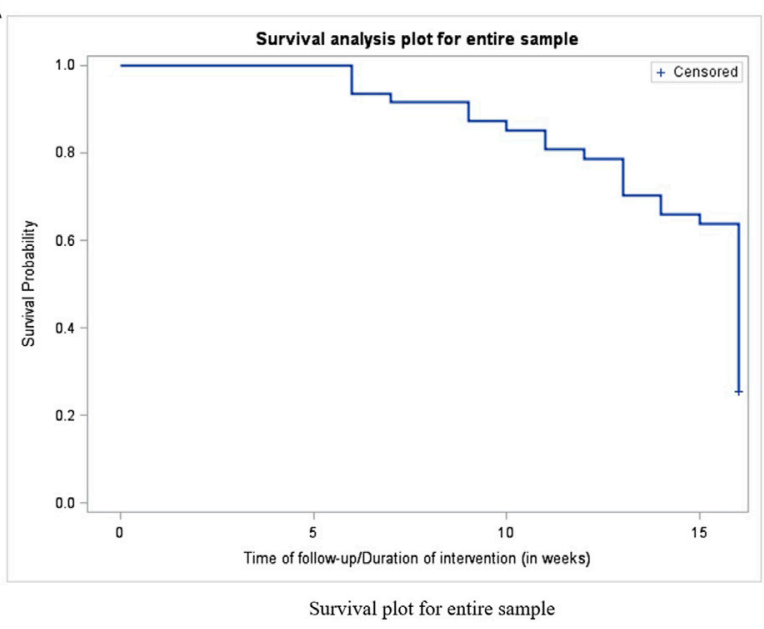

B

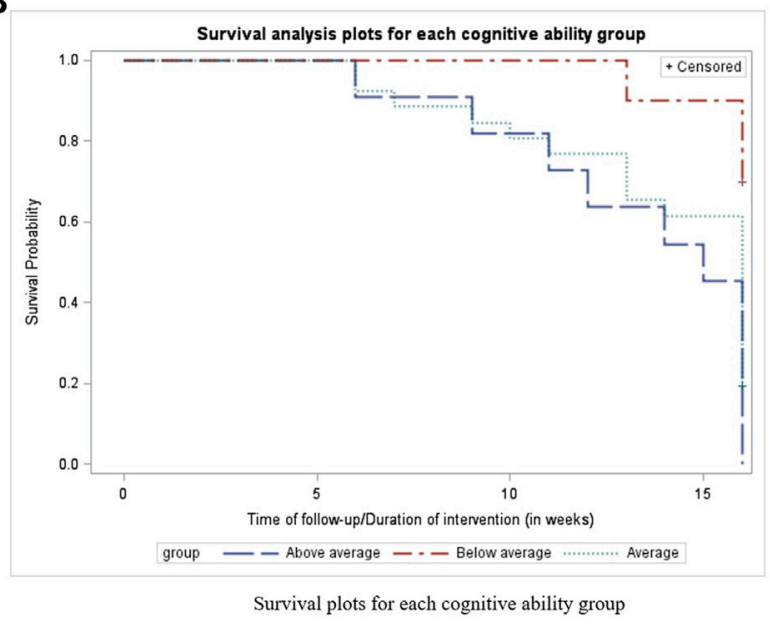

FIGURE 2 | Successfully achieved the crawling milestone within or at the end of the 16-week intervention using the SIPPC robot based on survival analysis: (A) Survival plot for entire sample. (B) Survival plots for each cognitive ability group.

did not complete the study and another had incomplete cognitive scores at baseline, resulting in 47 infants included in the final analyses. Table 1 contains mean age, birth weight, gestational age, and baseline Bayley III cognitive scores.

Although the mean MOCS subscale scores at baseline appeared different for infants in the "below average" cognitive ability group, and contained a higher degree of variability, none of the baseline scores were significantly different in any of the three subscales $(p=0.0523,0.6819$, and 0.3003 for subscales one, two and three, respectively) (Table 2 ).

\section{Time Differences}

The repeated measures ANOVA results demonstrate that within all three subscales, infants improved their scores at each time point ( $p<0.0001)$, with the exception of time " 2 " in Subscale 1 : Posture and Support ( $p=0.1355)$. Plots of the mean MOCS scores for each group over the five time points for each subscale are displayed in Figures 1A-C (Table 3).

\section{Group Differences}

Examination of group differences within subscale 1, "posture and support," reveal that only infants in the "below average" cognition group $\left(n_{2}=10\right.$, mean $\left.=5.3\right)$ were different from infants in the reference group, i.e., the "average" cognition group $\left(n_{3}=26\right.$, mean $=7.0$ ) with $p=0.0331$. No differences between group means were noted in subscale 2, "exploratory selection and progression," or in subscale 3, "mastery of propulsion."

\section{Interaction Effects}

In the analyses of subscale 1, the "posture and support" subscale of the MOCS, there was only one interaction effect between the "above average" cognition group $\left(n_{1}=11\right)$ at time " 2 " ( $\left.p=0.04\right)$ when compared to the "average" cognition group $\left(n_{3}=26\right.$, reference group). In the analyses of Subscale 2, "exploratory selection and progression" subscale of the MOCS, there was statistically significant interaction between the "below average" cognition group and all time points i.e., times " 2 ", “ 3 ”, “ 4 ”, and " 5 " when compared to the "average" cognition group $\left(n_{3}=26\right.$, reference group) and baseline time point (time " 1 "). Finally, in the analyses for subscale 3, "mastery of propulsion" subscale of the MOCS, there was no statistically significant interaction between group and time. We excluded all insignificant interaction terms from the final model for each subscale (Table 3).

Survival analysis revealed 35/47 (74.5\%) of the infant participants successfully achieved the crawling milestone by the end of the 16-week intervention. Comparing groups, $100 \%$ of the infants in the "above average" cognition group, $30 \%$ of the infants in the "below average" cognition group, and $80.8 \%$ infants in the of the "average" cognition group, successfully achieved the crawling milestone within or at the end of the 16-week intervention using the SIPPC robot (Table 4; 
Figure 2). Infants in both, the "above average" and "below average" cognition groups demonstrated significant differences $(p<0.0001)$ in their ability to achieve the crawling milestone when compared to the infants in the "average" cognition group within the 16-week intervention period. The odds of infants in the "above average" cognition group achieving crawling within the 16-week SIPPC intervention are $1.48(1.23,1.77)$ times higher than infants in the average group. The odds of infants in the "below average" cognition group achieving crawling the 16-week SIPPC intervention are 0.28 $(0.20,0.37)$ times lower than the infants in the average group.

\section{DISCUSSION}

The purpose of this study was to examine and evaluate the impact of cognitive ability of infants with and without CP on their ability to learn to use SIPPC robot. While baseline motor performance on the SIPPC (MOCS scores) between the three cognitive groups was not different, we found the rate of change of infants with lower cognitive ability differed from those with "average" or "above average" cognitive ability. Additionally, variability in the MOCS scores was higher in infants with lower cognitive ability when compared to infants with "average" or "above average" cognitive ability in all three subscales of the MOCS. These findings suggest that infants with lower cognitive ability took longer to master the motor skills involved in mobilizing the SIPPC robot than those with "average" or "above average" cognitive ability. Because the MOCS measures key movement strategies required to successfully learn to use the SIPPC robot, findings of this study corroborate results obtained from previous studies on the use of the SIPPC robot as an intervention for infants with or at risk of CP (Kolobe et al., 2015; Kolobe and Fagg, 2019).

In all three subscales of the MOCS, infants learned to use the SIPPC robot despite differences in cognitive status. Subscale one of the MOCS, "posture and support," measures how long an infant is able to maintain an upright head position. In this study, infants with "below average" cognitive ability were unable to maintain an upright head position for as long a duration as those with "average" cognitive ability. Because infants were challenged to maintain an upright head position in response to the presentation of toys that held their interest, this finding could suggest higher cognitive ability was linked to greater interest in the toys used. This in turn facilitated enhanced motor learning effort and ability. The positive interaction term also indicates infants with "above average" cognition learn to keep their heads up faster than those in the "average" range. This may also reflect early enhanced interest in interacting with toys.

MOCS subscale 2 (exploratory selection and progression) measures the frequency of arm and leg use, driven by motivation to get to the toys used during the SIPPC robot intervention sessions. Higher frequency of arm and leg movements indicate the infant"s efforts to move the SIPPC robot towards the toy, thus is a good indicator of movement coordination and problem-solving strategies. Our results indicate infants with "below average" cognitive ability did not only show fewer movement related problem-solving strategies with their arms and legs than infants with "average" cognitive ability, their learning curve was also slower. The lack of change in subscale two MOCS scores of infants in the below average cognitive group, especially over the first 9 weeks (3 time points) of intervention (Figure 1B) is consistent with other findings that support the association between delayed motor function and cognitive ability (Monteiro et al., 2010; Robert et al., 2013; Kolobe and Fagg, 2019). The gradual increase in MOCS scores during the last 3 weeks of training demonstrate children with below average cognitive ability may benefit from prolonged practice.

The MOCS subscale 3 (mastery of propulsion) measures the total number of trials required for an infant to develop goaloriented movements of the SIPPC robot before reaching the toy during intervention. This subscale also captures a degree of precision, error rate, and lack of effort. MOCS scores for infants with "below average" cognition changed at a slower rate than those with "average" or "above average" cognition. The slow rate of change (Figure 1C) indicates the presence of a delayed response time and a relatively higher and prolonged trialand-error rate, shown to be associated with cognitive ability (Adolph, 2008; Middleton and Schwartz, 2012).

The survival analysis results indicate a relationship between crawling and cognition. This finding is consistent with existing literature on crawling (Anderson et al., 2001; Adolph, 2008; Anderson et al., 2013) Although not the primary focus of this study, all infants with above average cognition attained independent crawling before or by the end of the SIPPC robot intervention compared to $30 \%$ in the group with below average cognition. The lower performance on the key movement requirement for moving the SIPPC sheds light on this relationship that has not been previously reported. It appears that ability to maintain head position to engage toys visually and the problem-solving needed to coordinate arm and leg movement to reach toys, which require cognitive ability, also play a major role in the SIPPC robot design and development.

Although the main variables of interest in this study were the cognitive and motor learning ability of infants, one major confounder is the motor ability level of the infant, which might also impact the learning outcomes using the SIPPC robot. The primary purpose of this study was to evaluate whether the SIPPC robot was designed to cater to infants with varying levels of cognitive ability. The Bayley III motor ability scores show that, among infants with "below average" cognitive ability, those with higher levels of motor ability still successfully achieved the crawling milestone. This finding is an indication that the SIPPC robot is well-matched to cognitive ability. We hypothesize failing to achieve the crawling milestone within 16 weeks of using the SIPPC robot can be explained by a poor motor ability level of the infants at baseline. The findings from this study will be instrumental in further modifying and refining the design of the SIPPC robot. Specifically, the SIPPC could better match the motor ability level of the infants by providing additional physical assistance. Future versions of the SIPPC robot can also be made more sensitive to smaller movements to help infants with lower muscle strength move the robot more easily. 


\section{CONCLUSION}

The findings in this study reveal the key movement strategies required to move the SIPPC robot, assessed by the MOCS, vary depending on the infants' cognition. While the results indicate a strong correlation between the infants' cognitive ability and motor learning ability (MOCS scores), it does not support a causal link between the two variables (infant cognition and motor learning ability). The findings also suggest that the design and development of the SIPPC robot allows successful use by infants with varying levels of cognitive ability, although infants with lower cognitive ability require a longer duration of training to successfully improve their motor skills. This study did not examine the contribution of gross motor development status to learning to use the SIPPC robot and to cognitive ability. Future studies looking at ability to learn to use the device among infants with varying levels of motor ability and cognitive are warranted.

\section{DATA AVAILABILITY STATEMENT}

The data analyzed in this study is subject to the following licenses/ restrictions: The dataset can be shared with the approval from OUHSC IRB and the PI of the study (TK who is the senior author for this article). Requests to access these datasets should be directed to TK, Hlapang-Kolobe@ouhsc.edu.

\section{REFERENCES}

Adolph, K. E. (2008). Learning to Move. Curr. Dir. Psychol. Sci. 17 (3), 213-218. doi:10.1111/j.1467-8721.2008.00577.x

Anderson, D. I., Campos, J. J., Anderson, D. E., Thomas, T. D., Witherington, D. C., Uchiyama, I., et al. (2001). The Flip Side of Perception-Action Coupling: Locomotor Experience and the Ontogeny of Visual-Postural Coupling. Hum. Mov Sci. 20 (4-5), 461-487. doi:10.1016/s0167-9457(01) 00063-x

Anderson, D. I., Campos, J. J., Witherington, D. C., Dahl, A., Rivera, M., He, M., et al. (2013). The Role of Locomotion in Psychological Development. Front. Psychol. 4, 440. doi:10.3389/fpsyg.2013.00440

Arneson, C. L., Durkin, M. S., Benedict, R. E., Kirby, R. S., Yeargin-Allsopp, M., Van Naarden Braun, K., et al. (2009). Prevalence of Cerebral Palsy: Autism and Developmental Disabilities Monitoring Network, Three Sites, United States, 2004. Disabil. Health J. 2 (1), 45-48. 2009/01/01/ 2009. doi:10.1016/j.dhjo.2008. 08.001

Bayley, N. (2006). Bayley Scales of Infant and Toddler Development. Third Edition. doi:10.1177/0734282906297199

Bayon, C., Raya, R., Lerma Lara, S., Ramirez, O., Serrano, J. I., and Rocon, E. (2016). Robotic Therapies for Children with Cerebral Palsy: A Systematic Review. Transl Biomed. 7. 01/01. doi:10.21767/2172-0479.100044

Bhasin, T. K., Brocksen, S., Avchen, R. N., and Van Naarden Braun, K. (2006). Prevalence of Four Developmental Disabilities Among Children Aged 8 years-Metropolitan Atlanta Developmental Disabilities Surveillance Program, 1996 and 2000. MMWR Surveill. Summ. 55 (1), 1-9. Jan 27.

Campbell, S. K., Kolobe, T. H., Wright, B. D., and Linacre, J. M. (2002). Validity of the Test of Infant Motor Performance for Prediction of 6-, 9- and 12-month Scores on the Alberta Infant Motor Scale. Dev. Med. Child. Neurol. 44 (4), 263-272. doi:10.1017/s0012162201002043

CDCdataforCP (2020). Data and Statistics for Cerebral Palsy. Available at: https:// www.cdc.gov/ncbddd/cp/data.html\#references.

de Vries, A. M., and de Groot, L. (2002). Transient Dystonias Revisited: a Comparative Study of Preterm and Term Children at 2 1/2 Years of Age. Dev. Med. Child. Neurol. 44 (6), 415-421. doi:10.1017/s0012162201002298

\section{ETHICS STATEMENT}

The studies involving human participants were reviewed and approved by The original study was reviewed by the University of Oklahoma IRB \#5120 and the secondary analysis used deidentified data. Written informed consent to participate in this study was provided by the participants' legal guardian/next of kin.

\section{AUTHOR CONTRIBUTIONS}

$\mathrm{AF}$ and TK designed and carried out the original experiments and data collection. RC and JR wrote the main text. HW, SJ, AF, TK edited the manuscript. RC, JR, SJ, and HW analyzed the data.

\section{ACKNOWLEDGMENTS}

This study was partially funded by the National Science Foundation (IIS-120863) and the Ann Taylor Endowment Fund. We will also like to thank the infants and parents who participated in the study, testers, Mustafa Ghazi, David Miller; and Caroline Wagner.

Ghazi, M. A., Nash, M. D., Fagg, A. H., Ding, L., Kolobe, T. H. A., and Miller, D. P. (2016). Novel Assistive Device for Teaching Crawling Skills to Infants. Springer Tracts Adv. Robotics, Field Serv. Robotics, 593-605. doi:10.1007/978-3-31927702-8_39

Hadders-Algra, M. (2000). The Neuronal Group Selection Theory: Promising Principles for Understanding and Treating Developmental Motor Disorders. Dev. Med. Child Neurol. 42 (10), 707-715. doi:10.1111/j.1469-8749.2000.tb00687.x

Hogan, N., and Krebs, H. I. (2004). Interactive Robots for Neuro-Rehabilitation. Restor Neurol. Neurosci. 22, 349-358.

Honeycutt, A., Dunlap, L., Chen, H., al Homsi, G., Grosse, S., and Schendel, D. (2004). Economic Costs Associated with Mental Retardation, Cerebral Palsy, Hearing Loss, and Vision Impairment. United State: 2003. Morbidity Mortality Weekly ReportMMWR.

Kolobe, T. H. A., and Fagg, A. H. (2019). Robot Reinforcement and Error-Based Movement Learning in Infants with and without Cerebral Palsy. Phys. Ther. 99 (6), 677-688. Jun 1. doi:10.1093/ptj/pzz043

Kolobe, T. H., Bulanda, M., and Susman, L. (2004). Predicting Motor Outcome at Preschool Age for Infants Tested at 7, 30, 60, and 90 Days after Term Age Using the Test of Infant Motor Performance. Phys. Ther. Dec 84 (12), 1144-1156. doi:10.1093/ptj/84.12.1144

Kolobe, H. A., Fagg, A., Pidcoe, P., and Williams, P. (2015). Effectiveness of Reward-And Error-Based Movement Learning in Enhancing Self-Initiated Prone Locomotion in Infants with or at Risk for Cerebral Palsy. Physiotherapy 101, e781-e782. doi:10.1016/j.physio.2015.03.3658

Krebs, H. I., and Hogan, N. (2006). Therapeutic Robotics: A Technology Push. Proc. IEEE 94 (9), 1727-1738. doi:10.1109/JPROC.2006.880721

McAdams, R. M., and Juul, S. E. (2011). Cerebral Palsy: Prevalence, Predictability, and Parental Counseling. NeoReviews 12 (10), e564-e574. doi:10.1542/neo.1210 -e564

Middleton, E. L., and Schwartz, M. F. (2012). Errorless Learning in Cognitive Rehabilitation: A Critical Review. Neuropsychological Rehabil. 22 (2), 138-168. 2012/04/01. doi:10.1080/09602011.2011.639619

Miller, D. P., Fagg, A. H., Ding, L., Kolobe, T. H., and Ghazi, M. A. (2015). "Robotic Crawling Assistance for Infants with Cerebral Palsy," in Proceedings of AAAI Workshop on Artificial Intelligence Applied to Assistive Technologies and Smart Environments. January. 
Monteiro, C. B. d. M., Jakabi, C. M., Palma, G. C. d. S., Torriani-Pasin, C., and Meira Junior, C. D. M. (2010). Aprendizagem Motora Em Crianças Com Paralisia Cerebral. J. Hum. Growth Dev. 20 (2), 250-262. doi:10.7322/jhgd.19963

NINDSWebsite (2019). Cerebral Palsy: Hope through Research. Available at: https://www.ninds.nih.gov/Disorders/Patient-Caregiver-Education/HopeThrough-Research/Cerebral-Palsy-Hope-Through-Research\#3104_2.

Novak, I., Mcintyre, S., Morgan, C., Campbell, L., Dark, L., Morton, N., et al. (2013). A Systematic Review of Interventions for Children with Cerebral Palsy: State of the Evidence. Dev. Med. Child. Neurol. 55 (10), 885-910. doi:10.1111/ dmcn.12246

Oskoui, M., Coutinho, F., Dykeman, J., Jetté, N., and Pringsheim, T. (2013). An Update on the Prevalence of Cerebral Palsy: a Systematic Review and Meta-Analysis. Dev. Med. Child. Neurol. 55 (6), 509-519. doi:10.1111/dmcn.12080

Peter, R The Definition and Classification of Cerebral Palsy. Dev. Med. Child. Neurol. 2007;49(s109):1-44. doi:10.1111/j.1469-8749.2007.00001.x

Raya, R., Rocon, E., Urendes, E., Velasco, M. A., Clemotte, A., and Ceres, R. (2015). "Assistive Robots for Physical and Cognitive Rehabilitation in Cerebral Palsy," in Intelligent Assistive Robots: Recent Advances in Assistive Robotics for Everyday Activities. Editors S. Mohammed, J. C. Moreno, K. Kong, and Y. Amirat (Springer International Publishing), 133-156. doi:10.1007/978-3-319-12922-8_5

Robert, M. T., Guberek, R., Sveistrup, H., and Levin, M. F. (2013). Motor Learning in Children with Hemiplegic Cerebral Palsy and the Role of Sensation in ShortTerm Motor Training of Goal-Directed Reaching. Dev. Med. Child. Neurol. 55 (12), 1121-1128. doi:10.1111/dmcn.12219

Rule, B. A. (2010). Motor Strategies Implemented by Infants Using the Self-Initiated Prone Progression Crawler.

Sadowska, M., Sarecka-Hujar, B., and Kopyta, I. (2020). Cerebral Palsy: Current Opinions on Definition, Epidemiology, Risk Factors, Classification and Treatment Options. Ndt 16, 1505-1518. doi:10.2147/ndt.s235165
Shonkoff, J. P., and Meisels, S. (2000). Handbook of Early Childhood. Intervention. Stadskleiv, K. (2020). Cognitive Functioning in Children with Cerebral Palsy. Dev. Med. Child. Neurol. 62 (3), 283-289. doi:10.1111/dmcn.14463

Turk, M. A. (2009). Health, Mortality, and Wellness Issues in Adults with Cerebral Palsy. Dev. Med. Child. Neurol. 51 (Suppl. 4), 24-29. doi:10.1111/j.1469-8749. 2009.03429.x

van Gorp, M., Hilberink, S. R., Noten, S., Benner, J. L., Stam, H. J., van der Slot, W. M. A., et al. (2020). The Epidemiology of Cerebral Palsy in Adulthood: A Systematic Review and Meta-Analysis of the Most Frequently Studied Outcomes. Arch. Phys. Med. Rehabil. 101 (6), 1041-1052. doi:10.1016/j. apmr.2020.01.009

Conflict of Interest: The authors declare that the research was conducted in the absence of any commercial or financial relationships that could be construed as a potential conflict of interest.

Publisher's Note: All claims expressed in this article are solely those of the authors and do not necessarily represent those of their affiliated organizations, or those of the publisher, the editors and the reviewers. Any product that may be evaluated in this article, or claim that may be made by its manufacturer, is not guaranteed or endorsed by the publisher.

Copyright (c) 2022 Chandrashekhar, Wang, Rippetoe, James, Fagg and Kolobe. This is an open-access article distributed under the terms of the Creative Commons Attribution License (CC BY). The use, distribution or reproduction in other forums is permitted, provided the original author(s) and the copyright owner(s) are credited and that the original publication in this journal is cited, in accordance with accepted academic practice. No use, distribution or reproduction is permitted which does not comply with these terms. 